\title{
Commentaire
}

\section{Le SRADDET, un « outil » de planification et de développement territorial durable dans les régions françaises?}

\author{
Jean-Paul Carrière ${ }^{1 凶}$
}

${ }^{1}$ UMR CNRS 7324 CITERES, Université de Tours, France

\begin{abstract}
Résume. La Réforme régionale française a suscité de nombreuses réactions publiques relatives au nouveau découpage des régions. Mais, dans cet article, on s'interroge sur la portée de l'un des aspects de la réforme régionale française, passé quelque peu inaperçu, à savoir la création du Schéma Régional d'Aménagement, de Développement Durable et à l'Egalité des Territoires (SRADDET). Selon les termes de la Loi NOTRe de 2015, ce nouveau document de planification stratégique à l'échelle régionale a vocation à devenir la matrice de l'ensemble des documents de planification territoriale du fait de son double caractère prescriptif et opposable. Mais pour autant, peut-il prétendre devenir l'instrument d'un véritable développement durable de portée régionale ? et quelles seraient les conditions permettant de répondre à cette finalité ? Des premiers éléments de réponse à ces questions sont proposés, en s'appuyant principalement, mais de façon non exclusive, sur l'avant-projet de schéma en cours d'adoption dans la Région Centre - Val de Loire.
\end{abstract}

Mots-clés : Schéma Régional d'Aménagement, de Développement Durable et à l'Egalité des Territoires (SRADDET), Développement territorial durable, France, Région Centre-Val de Loire

\begin{abstract}
The French regional reform aroused public reactions mainly on the issue for the new delimitation of regions. In this article, we question the scope of one particular aspect of the reform, which has gone somewhat unnoticed, namely the creation of the Regional Scheme of Planning, Sustainable Development and Territorial Equality. Under the terms of the NOTRe Law of 2015, this new regional strategic planning document aims to become the matrix of all territorial planning documents due to its dual nature, both prescriptive and enforceable. However, can it claim to become the instrument of genuine regional sustainable development? Moreover, what would be the conditions to meet this purpose? We propose some elements of answer to these questions, relying mainly - but not exclusively - on the preliminary draft scheme of the Centre Val de Loire region.
\end{abstract}

Keywords : Sustainable Development Regional Plan for territorial equality (SRADDET), sustainable territorial development, France, Centre-Val de Loire Region

\footnotetext{
CORRESPONDENCE:

$\triangle 35$ Allée Ferdinand de Lesseps, 37200 Tours, France.

@ carriere@univ-tours.fr
} 


\section{Introduction}

Qualifié par certains de "Big Bang territorial » (Torre et Bourdin, 2015), le profond bouleversement de l'architecture territoriale française survenu en France dans la première moitié de la décennie 2010 avec l'adoption d'une part de la loi de 2010 (révisée en 2014) sur la Modernisation de l'Action Publique Territoriale et l'Affirmation des Métropoles (Loi Maptam) et d'autre part de la Loi de 2015 sur la Nouvelle Organisation Territoriale de la République (Loi NOTRe) a eu de profondes conséquences sur l'organisation politico-administrative du territoire français. C'est ainsi que sont apparues les métropoles, de nouveaux périmètres régionaux et de nouvelles formes d'intercommunalités, tandis que les Départements et les Régions voyaient leurs compétences profondément modifiées (cf. Annexe 1).

En ce qui concerne les Régions, trois modifications majeures sont à relever, à savoir la diminution de leur nombre, passé en France métropolitaine de 22 à 13, avec pour corollaire :

- Un agrandissement très important du périmètre de certaines d'entre elles et un changement de capitale.

- La suppression (comme pour les Départements) de la clause de compétences générales avec pour contrepartie la définition par la Loi de compétences spécifiques, en particulier l'aménagement du territoire, et de compétences partagées avec les autres collectivités,

- Et enfin le remplacement de l'ancien Schéma Régional d'Aménagement et de Développement du Territoire (SRADDT), document stratégique sans valeur prescriptive, par le Schéma régional d'Aménagement, de Développement Durable et d'Égalité des Territoires (SRADDET), désormais prescriptif et opposable aux tiers, et devenant en quelque sorte la « matrice » à la fois des documents de planification sectorielle et des schémas élaborés par les autres échelons de pouvoir local qui devront s'y conformer.

Ce dernier volet de la réforme est passé relativement inaperçu, le débat public s'étant avant tout focalisé sur la question des délimitations des Régions. Pour autant, ce n'est pas une modification mineure, car elle consacre le rôle majeur donné aux nouvelles Régions en matière de cohésion territoriale.

En aménagement du territoire, il est commun de distinguer trois types de projet territorial : le projet « conceptuel » et utopique (par exemple le projet de la cité-jardin d'E. Howard, 1898), le projet opérationnel d'aménagement physique (projet $\mathrm{d}^{\prime}$ infrastructure, ville nouvelle, etc...) d'un espace particulier, et le projet stratégique fondé sur une «vision spatiale » et un diagnostic prospectif d'un territoire. Se voulant souple, flexible et évolutif, le SRADDET relève directement de cette dernière 
approche du projet territorial, et répond, comme dans beaucoup d'autres pays à travers le monde, aux évolutions en cours, marquées à la fois par les incertitudes résultant de la globalisation, à l'influence accrue des conceptions et pratiques anglosaxonnes de l'aménagement, à la remise en question de l'aménagement ordonnateur pour cause de néolibéralisme, et au développement des démarches plus ou moins affirmées de démocratie participative.

Cette «petite révolution » dans la répartition des compétences et dans la hiérarchie des pouvoirs locaux en matière d'aménagement du territoire n'est pas sans poser un certain nombre de questions auxquelles nous tenterons dans cet article d'apporter des éléments de réponses, en nous appuyant principalement, mais de façon non exclusive, sur le cas de la Région Centre-Val de Loire.

Le questionnement principal porte sur l'aptitude du SRADDET à devenir un véritable outil du développement territorial durable à l'échelle régionale. Pour y répondre, nous commencerons par présenter aux lecteurs, qui ne sont pas nécessairement au fait de l'actualité régionale française, le SRADDET et les grands axes de la réforme des régions réalisée ces dernières années en France. Dans un second temps, nous évoquerons à titre illustratif le cas du SRADDET de la Région Centre - Val de Loire, sachant que celui-ci, au moment (mars 2019) où nous rédigeons cet article, est encore en cours d'élaboration et n'est pas définitivement adopté ${ }^{2}$ puis nous tenterons de mieux cerner la portée et les limites du SRADDET, en s'interrogeant sur la résurgence des grands principes de l'aménagement du territoire « à la française » qu'il est supposé permettre, pour enfin en évaluer la portée à l'aune des principes du développement territorial durable (DTD). La conclusion nous amènera à identifier les défis que les Régions devraient relever au moment d'adopter leurs schémas dans une perspective de DTD.

\section{Le SRADDET, une innovation institutionnelle pour faire de la Région le "chef de file" de l'aménagement du territoire}

Comme indiqué plus haut, le contexte de la création du SRADDET est celui d'une réforme territoriale, qui se veut ambitieuse et induisant de profondes modifications de l'architecture territoriale de la France. Trois motifs principaux ont été mis en avant pour la justifier :

- Simplifier et clarifier une organisation territoriale, souvent qualifiée de "millefeuilles", du nom d'une pâtisserie, bien connue en France, constituée d'un empilement de fines couches de pâte feuilletée...

\footnotetext{
2 Comme dans toutes les Régions françaises
} 
- Doter les régions françaises, jugées trop petites par rapport à leurs concurrentes européennes (en particulier allemandes), d'une "taille critique" et les rendre plus compétitives dans la compétition mondiale. ${ }^{3}$

- Réaliser des économies d'échelle et rationnaliser la dépense publique, notamment à travers la redistribution des compétences entre chaque niveau de pouvoir local.

C'est au nom de ces motifs qu'ont été redécoupées les Régions (Figure 1), redéfinies les structures intercommunales et revue la répartition des compétences entre les différents niveaux de pouvoir local.

L'énoncé de ces motifs et les décisions qui en ont découlé à travers la rédaction de la Loi NOTRe ont suscitées de nombreuses controverses, et force est de constater que le débat est resté, et reste toujours, vif dans le pays quant à la pertinence des arguments avancés. Le scepticisme semble dominer parmi les analyses de la réforme proposées par nombre d'acteurs ou de chercheurs en science régionale (Torre et Bourdin, 2015). Toutefois, si nous partageons avec ces analyses nombre d'interrogations, le but de cet article n'est pas de revenir sur la pertinence des justifications avancées par le gouvernement à l'origine de la réforme, mais plutôt de tenter une évaluation ex-ante des effets de la création du SRADDET, qui apparaît comme l'une des innovations importantes inscrites dans la loi entrant désormais en vigueur, puisque les SRADDET doivent être adoptés dans toutes les Régions, exception faite des cas particuliers de l'Île de France, de la Corse des Régions d'Outre-mer, fin 2019 pour une mise en œuvre dès 2020.

Selon l'esprit de la Loi, le SRADDET a pour fonction de proposer une vision unifiée des enjeux et des objectifs d'aménagement régional, en vue d'un développement durable et équilibré des territoires qui la composent : métropoles", villes petites et moyennes, intercommunalités et territoires ruraux. On voit à travers cela le rôle central réservé par le législateur aux Régions en matière de planification et d'aménagement du territoire. En effet le SRADDET se présente comme un document de planification qui fixe des objectifs à moyen et long terme (2025 et 2050), en étant à

\footnotetext{
${ }^{3} C^{\prime}$ est pourquoi le nombre des Régions en France métropolitaine a été ramené de 22 à 13, par fusion des anciennes, avec cependant cinq exceptions, dont la Région Centre - Val de Loire, ayant conservé leur périmètre antérieur. Du fait de ces fusions, certaines Régions françaises ont désormais une taille supérieure à de nombreux États européens, que ce soit en termes de population ou de superficie. Par exemple la Région Nouvelle Aquitaine, issue de la fusion de trois anciennes Régions, est aujourd'hui plus grande que l'Autriche!
}

\footnotetext{
4 Parallèlement au redécoupage des Régions, ont été créées sous l'effet de la loi MAPTAM 22 métropoles au sein des plus grandes agglomérations françaises, ce qui n'est pas sans effet sur l'élaboration des SRADDET, puisque la loi NOTRe dispose que les métropoles doivent être obligatoirement consultées pour avis avant l'adoption définitive des schémas.
} 
la fois prescriptif pour les collectivités infrarégionales (Départements, intercommunalités, communes) et intégrateur de tous les autres schémas régionaux relatifs à des stratégies sectorielles ${ }^{5}$. Le SRADDET se définit donc comme un document de planification stratégique et de cohérence territoriale obligatoire, opposable aux tiers, sans que l'on puisse pour autant parler d'un retour à une forme de planification rigide, comme on le verra par la suite.

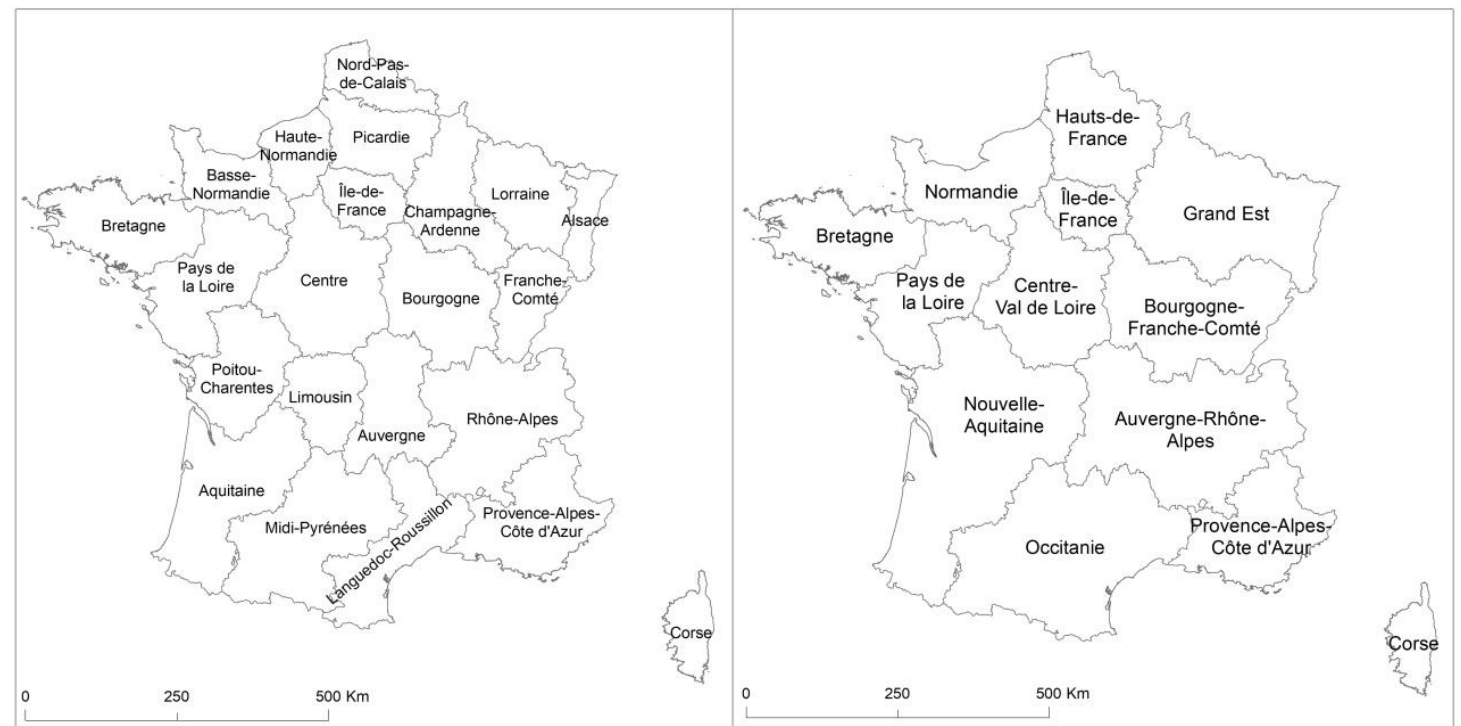

Figure 1. Les anciennes (à gauche) et les nouvelles (à droite) limites des régions françaises (hors régions ultramarines)

Tout en respectant les règlements et projets d'intérêt général nationaux ou européens, notamment les directives de l'UE, le SRADDET devra s'imposer au niveau local (notion d'opposabilité). La loi NOTRe le place au sommet de la hiérarchie des normes de documents de planification territoriale. Ceci signifie que les futurs documents locaux de planification et d'urbanisme, tels que les Plans Locaux d'Urbanisme (PLU) déterminant l'affectation des sols, ou les Schémas de Cohérence Territoriale (SCoT) à certains égards comparables aux PUG roumains, ou encore les chartes des Parcs Naturels Régionaux devront prendre en compte les objectifs et les règlements de la Région en matière d'aménagement du territoire. Les documents d'urbanisme déjà adoptés et en cours d'application devront, quant à eux, être mis en compatibilité a posteriori, lors de leur révision. A travers le SRADDET, la Loi NOTRe

5 Comme par exemple, le Schéma régional de développement économique, d'innovation et d'internationalisation, le Schéma régional de l'enseignement supérieur, de la Recherche et de l'innovation, le Schéma régional de cohérence écologique, le Plan régional de prévention et de gestion des déchets, etc.) 
institue de fait un cas unique de prescriptivité d'une collectivité sur une autre, puisque les actes des collectivités locales devront respecter les règles générales définies au niveau régional. Toutefois, il en découle une situation quelque peu paradoxale, puisque la Constitution de la République Française interdit à une collectivité d'imposer des charges supplémentaires à une autre, ce que la loi NOTRe cherche à contourner en prévoyant un principe de "co-construction" du SRADDET, par concertation entre les différents niveaux de collectivité, comme on le verra plus loin.

Le SRADDET a pour finalité de fixer des objectifs de moyen et long terme dans trois domaines précis: l'aménagement du territoire régional, l'organisation des transports à l'échelle régionale, et la gestion de l'environnement. Il concerne obligatoirement 12 thèmes, à savoir :

- L'équilibre et l'égalité des territoires,

- L'implantation des différentes infrastructures d'intérêt régional,

- Le désenclavement des territoires ruraux,

- L'habitat,

- La gestion économe de l'espace,

- L'intermodalité et le développement des transports,

- La maîtrise et la valorisation de l'énergie,

- La lutte contre le changement climatique,

- La pollution de l'air,

- La protection et la restauration de la biodiversité,

- La prévention et la gestion des déchets.

Cependant, cette liste n'est pas limitative. En dehors de ces thèmes obligatoires, le SRADDET peut également porter sur tout domaine contribuant à l'aménagement du territoire régional. L'ensemble du projet est soumis à enquête publique.

L'architecture du Schéma reste, somme toute, assez classique, et comporte :

- Un rapport composé de deux parties, la première présentant un diagnostic des enjeux relevant des domaines de compétence de la Région, à partir d'un état des lieux du territoire régional, la seconde énonçant la stratégie et les objectifs de moyen et long terme opposables aux tiers.

- Un fascicule divisé en chapitres thématiques déclinant les objectifs en règles générales que devront prendre en compte les autres collectivités et groupements à travers leurs actes et choix d'investissement. Ce document devra également préciser les mesures d'accompagnement que la Région souhaite mettre en place pour aider ses partenaires publics et privés à atteindre les objectifs, ainsi que des indicateurs de suivi et d'évaluation. Les 
règles générales, seules à être prescriptives, s'adressent aux plans et programmes locaux et s'inscrivent dans un rapport de compatibilité avec les documents de rang inférieurs, ces derniers ne pouvant prendre de disposition à l'encontre de ces règles. Ces dernières peuvent être complétées par des recommandations, elles non contraignantes.

- Une carte synthétique aux 150 millièmes, illustrant les objectifs du Schéma.

- Des annexes (diagnostics thématiques, évaluation des incidences environnementales du SRADDET, état des lieux de la prévention des déchets, diagnostic des continuités écologiques retenues pour constituer la trame verte et bleue régionale, etc.)

La Loi impose le principe d'une co-élaboration transversale avec les acteurs et partenaires concernés, de façon à rendre acceptable la prescriptivité du Schéma. Elle prévoit à cette fin l'organisation d'une Conférence Territoriale de l'Action Publique (CTAP), pour le choix des grands objectifs. Sont autour de la table de plein droit toutes les collectivités infrarégionales, le Conseil Économique, Social et Environnemental Régional (CESER), les organismes consulaires (Chambres de Commerce, etc.), mais peut aussi être consulté tout autre organisme identifié par la Région et la population, à travers l'organisation de forums ouverts à tous, dans une optique de démocratie participative.

\section{Le cas du SRADDET « à $360^{\circ}$ » de la Région Centre - Val de Loire}

Selon les termes mêmes du Président de la Région CVL, François Bonneau, la stratégie d'aménagement se fonde sur une "vision globale et unifiée du territoire régional, à 360 degrés". Le projet inclut des objectifs à moyen terme (2025/2030) et des objectifs à plus long terme (2050), en vue d'un aménagement et du développement durable de la Région. La stratégie, selon François Bonneau, "a pour ambition d'assurer un développement équilibré et complémentaire de tous les territoires de notre région". À cette fin, un très large processus de concertation avec tous les acteurs concernés, publics et privés, y compris les régions voisines, et citoyens, est engagé en vue d'élaborer une stratégie d'aménagement "non imposée et partagée".

À travers, l'invocation d'un "schéma à $360^{\circ}$ ", c'est un projet collectif qui sera proposé fin 2019 à l'assemblée délibérative, le Conseil Régional, englobant à la fois toutes les dimensions du développement durable et tous les territoires de la Région. Pour mieux en appréhender la portée des objectifs et des règles, une brève présentation du contexte régional est un préalable nécessaire.

\subsection{Centre - Val de Loire, des dynamiques favorables et un territoire fragmenté}

La Région Centre - Val de Loire (ou Région CVL) est la plus petite des Régions françaises du continent européen sur le plan démographique, exception faite de la 
Corse. Elle rassemblait en 2017 près de 2578000 habitants. En revanche, forte de ses quelque $40000 \mathrm{Km}^{2}$, elle se situe au $4{ }^{\text {ème }}$ rang national pour sa superficie et s'étend du sud-est du Bassin Parisien aux confins septentrionaux du Massif Central. Région à faible densité (66 habitants/ $\mathrm{Km}^{2}$ en 2014 - source : INSEE), elle regroupe des entités paysagères diverses et contrastées, parmi lesquelles se distinguent la Vallée de la Loire Moyenne, inscrite sur près de $200 \mathrm{Km}$ au patrimoine mondial de l'Humanité, du fait de la qualité de ses paysages et de son patrimoine historique et culturel exceptionnel (les châteaux de la Loire), les grandes plaines céréalières du nord régional, comme la Beauce, les zones humides émaillées de forêts, de landes et d'étangs, plus au sud, comme la Brenne et la Sologne, des zones de côteaux et de reliefs légers, et la plus vaste forêt domaniale de France, la forêt d'Orléans. Cette juxtaposition d'entités paysagères lui permet de réunir des milieux naturels particulièrement riches en termes de biodiversité.

Région, dont l'appareil productif reste fortement marqué par les grandes cultures (céréales, Colza, betterave sucrière...), Centre - Val de Loire conserve de fortes spécificités industrielles, ce qui la rend attractive pour les investissements directs étrangers (30\% des emplois industriels). Elle se range parmi les premières régions françaises dans des secteurs tels que l'industrie cosmétique (1er rang national), l'industrie pharmaceutique ( $2^{\text {ème }}$ rang), la production de caoutchouc industriel (2 $\left.2^{\mathrm{ème}} \mathrm{rang}\right)$, la production électrique (1 ${ }^{\text {ère }}$ région électro-nucléaire). Mais à l'instar des autres régions françaises, son économie est aujourd'hui très largement tournée vers le secteur tertiaire, avec une forte incidence du tourisme ou du numérique en pleine expansion. L'économie de la connaissance voit son développement tiré par les deux universités de Tours (plus de 30000 étudiants) et d'Orléans (près de 20000 ), et de grands établissements nationaux de recherche. Elle dispose enfin d'un réseau d'infrastructures assez dense, et occupe une place de carrefour dans les grands flux de mobilité nationaux, notamment grâce au TGV desservant Tours, tandis qu'Orléans, la capitale régionale, non raccordée au réseau TGV, est cependant à une heure de Paris.

Région au niveau de vie globalement élevé, car faisant partie des cinq régions françaises les plus riches, Centre - Val de Loire connaît une dynamique démographique positive $\mathrm{du}$ fait d'une attractivité avérée pour des populations venant des régions voisines, principalement de l'île de France. Les entrées de nouveaux habitants profitent principalement au nord régional, les "franges franciliennes"', et à la vallée de la Loire et son tissu urbain. Cela ne fait que traduire une réalité marquée par une fragmentation en trois systèmes territoriaux distincts, elle-même source de fragilité de la cohésion territoriale et sociale du territoire régional.

${ }^{6}$ Terme désignant les territoires limitrophes de la Région parisienne, l'Île de France 
En effet, les différents territoires et bassins de vie constitutifs de la région se répartissent en trois grands ensembles relativement homogènes en matière démographique, d'emplois, et de flux de personnes : le nord régional, largement soumis aux influences franciliennes et pour partie intégré dans l'aire urbaine de Paris, l'axe ligérien formé par la vallée de la Loire et son chapelet de villes, dont les dynamiques démographiques, d'emplois et de revenus sont les plus favorables, et enfin le Berry, territoire historique au sud de la région, plus rural et confronté à des dynamiques récessives en termes de vieillissement et de désertification démographiques et à un niveau plus élevé de chômage, malgré une ancienne tradition industrielle encore présente, mais en déclin. La région reste maillée par une armature urbaine relativement équilibrée comprenant deux grandes aires urbaines ${ }^{7}$, sises sur les bords de la Loire, exerçant des fonctions de centralité à l'échelle régionale, Tours (491 000 habitants) et Orléans (430 000 habitants), 6 agglomérations de taille moyenne, et 24 pôles de centralité, autrement dit de villes de plus de 5000 habitants polarisant les bassins de vie infra-régionaux. Les agglomérations de Tours et Orléans, qui ne recouvrent pas l'intégralité de leurs aires urbaines, ont récemment acquis le statut de "métropole".

Ce maillage est vu comme une "chance" par le rapport du SRADDET dans sa version provisoire (Région Centre - Val de Loire 2018b), ce qui ne doit pas cependant occulter le paradoxe et double risque d'une fracture territoriale, notamment entre les deux sous-ensembles du nord régional et du corridor ligérien (la vallée de la Loire), et d'une métropolisation «incomplète», du fait de la concurrence et du manque de coopération entre deux «petites » métropoles au regard de leurs rivales nationales et européennes, plus attractives. Les deux métropoles peinent en effet à se projeter à l'échelle régionale et à jouer ensemble un véritable rôle d'entraînement au-delà de leur aire d'influence immédiate.

C'est cette réalité territoriale, présentée ici de façon sommaire, qui explique une stratégie d'aménagement à l'horizon 2030, (Figure 2). Le projet spatial que traduit cette carte vise à valoriser les spécificités et les atouts de chacun des trois grands ensembles régionaux, à tirer parti du maillage de l'armature urbaine en vue d'un développement complémentaire de tous les territoires, urbains comme ruraux, à renforcer les synergies entre ces mêmes territoires par des coopérations renforcées, et à développer des liens avec les régions limitrophes.

\footnotetext{
7 Au sens de l'Institut National de la Statistiques et des Études Économiques, une aire urbaine est un ensemble de communes, d'un seul tenant et sans enclave, constitué par un pôle urbain (unité urbaine) de plus de 10000 emplois, et par des communes rurales ou unités urbaines (couronne périurbaine) dont au moins $40 \%$ de la population résidente ayant un emploi travaille dans le pôle ou dans des communes attirées par celui-ci.
} 


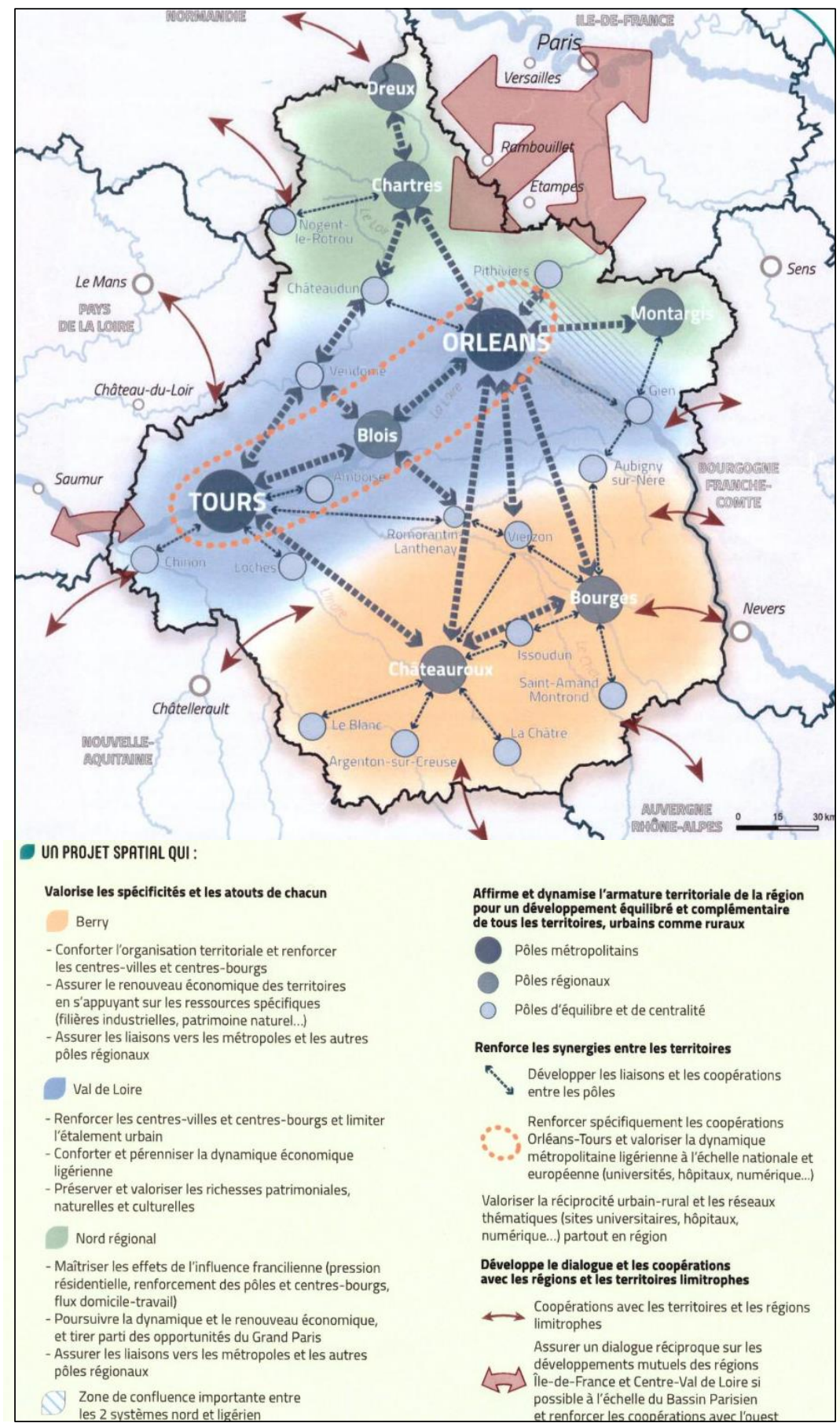

Figure 2. Projet spatial de la Région CVL à l'horizon 2030

Source : Région Centre -Val de Loire (2018a) 


\subsection{Le SRADDET de la Région CVL, orientations et contenu. ${ }^{8}$}

Les perspectives de long terme esquissées dans le projet de schéma régional permettent de fixer des objectifs très ambitieux, voire d'envisager certaines pistes qui peuvent apparaître aujourd'hui comme utopiques, mais relevant d'une approche volontariste.

Conformément aux exigences de la Loi NOTRe, le SRADDET de la Région CVL intègre complètement le Schéma Régional de Cohérence Écologique de 2014, le Schéma Régional Climat Air Energie (SRCAE) de 2012, et le Plan Régional de Prévention et de Gestion des Déchets (PRPGD) de 2018, qui définissaient déjà les objectifs de la Région dans les domaines concernés. Le projet de SRADDET est également construit, comme le veut la Loi, en cohérence avec le Schéma Régional de Développement Économique, d'Innovation et d'Internationalisation (SRDEII adopté en 2017) , avec la Stratégie régionale tourisme, la Stratégie numérique, le Schéma Régional de l'Enseignement Supérieur, de la Recherche et de l'Innovation (SRESRI, également adopté en 2017), et avec d'autres documents plus spécialisés qui définissent les priorités de politique régionale dans leurs domaines respectifs. Par exemple en matière d'enseignement supérieur, le SRESRI prévoit une forte croissance du nombre d'étudiants accueillis dans les universités régionales, et le SRADDET reprend cet objectif avec un effectif de 80000 étudiants à l'horizon 2030.

Le SRADDET de la Région CVL se décline en 4 orientations et 20 objectifs et 47 règles. Si les orientations stratégiques sont définies sous des intitulés extrêmement larges et en eux-mêmes peu précis, les objectifs, sans imposer de fortes contraintes aux collectivités locales et aux autres acteurs de l'aménagement, indiquent les limites que ceux-ci ne devront pas transgresser dans leurs partis d'aménagement (par exemple : ne pas planifier une action susceptible de porter atteinte à la qualité de $l^{\prime}$ eau, dès lors que le SRADDET a pour objectif $\left(\mathrm{N}^{\circ} 17\right)$ de préserver celle-ci). Mais ce sont les règles qui donnent au SRADDET son caractère réellement prescriptif, puisque les plans et programmes des acteurs locaux ne pourront inclure des dispositions allant à l'encontre ou remettant en cause ces règles.

La première orientation stratégique sous le titre: "Des femmes et des hommes acteurs $d u$ changement, des villes et des campagnes en mouvement permanent pour une démocratie renouvelée" regroupe 4 objectifs (Objectifs 1 à 4$)^{9}$ :

- Faire vivre la démocratie permanente dans les territoires (objectif 1),

\footnotetext{
8 Ce paragraphe reprend nombre d'éléments de synthèse issus de l'avis du sur le projet provisoire. Cet avis a été adopté en session plénière par les membres du Conseil Économique, Social et Environnemental de la Région CVL, le 17 décembre 2018.

${ }^{9}$ Dans les développements qui suivent, nous ne reprenons que les éléments qui nous paraissent les plus saillants dans l'énoncé des objectifs. On ne peut donc considérer cette présentation comme ayant un caractère d'exhaustivité.
} 
- Renforcer les coopérations, notamment intercommunales, et le dialogue entre les territoires (objectif 2), le but étant d'aller vers des échelles de territoires plus vastes prenant en compte la réalité du territoire vécu par les habitants,

- Faire vivre des réseaux thématiques de réflexion innovants (objectif 3) pour permettre les échanges de connaissance et les retours d'expériences,

- Renforcer les coopérations avec les régions voisines, en particulier la Région parisienne, mais aussi les autres régions limitrophes (objectif 4).

La seconde orientation : Affirmer l'unité et le rayonnement de la région Centre-Val de Loire par la synergie de tous ses territoires et la qualité de vie qui la caractérise" intègre 5 objectifs (5 à 9). Elle porte l'ambition de développer un urbanisme et un habitat durable centré autour de ses pôles urbains et de ses centres-bourgs (objectif 5), dynamisés et reliés par un système de mobilité inclusif et multimodal. En clair, il s'agit de freiner, et, à terme de mettre fin au mouvement de périurbanisation et à l'étalement urbain (diviser par 2 la consommation d'espaces agricoles, naturels et forestiers d'ici 2025 et tendre vers zéro artificialisation nette d'espaces agricoles naturels et forestiers en 2030).

L'objectif 6 vise plusieurs cibles et notamment celle de réduire les consommations énergétiques finales du territoire régional par rapport à 2014 de $43 \%$ d'ici à 2050, dans le but de devenir une région couvrant ses besoins énergétiques à $100 \%$ par les énergies renouvelables et de récupération. Est également affirmée la volonté de réduire de $100 \%$ les émissions de gaz à effet de serre d'origine énergétique entre 2014 et 2050.

Concernant les transports et les mobilités (objectif 7), le SRADDET propose de faciliter tous types de report modaux de la voiture individuelle vers d'autres modes plus durables, de sauvegarder un réseau ferroviaire du quotidien dense et performant, de concevoir une politique de tarification, billettique et de distribution articulée avec les principes de desserte, simple et cohérente à l'échelle régionale, et d'encourager les innovations et les expérimentations, notamment en matière $\mathrm{d}$ 'hydrogène, - etc. Pour le numérique, on ne peut que souscrire au souhait de couvrir l'intégralité du territoire par le THD à l'horizon 2025 pour permettre une «inclusion numérique universelle».

En matière de santé la Région se fixe pour objectif de passer entre 2018 et 2025 de 950 à 1500 professionnels de santé dans les structures d'exercice regroupé (dont 450 médecins généralistes en 2025 [280 en 2018]), d'atteindre une densité de médecins généralistes « tous modes d'exercice » de 1,3 pour 1000 habitants en 2030, ce qui est ambitieux au regard de la situation présente marquée par un fort processus de désertification médicale (densité inférieure à 1). 
L'orientation 3 propose une stratégie pour "Booster la vitalité de l'économie régionale en mettant nos atouts au service d'une attractivité renforcée" (Objectifs 10 à 15)

L'objectif 10, consacré au tourisme et au développement économique, souligne la nécessité de développer un marketing territorial propre à démarquer la région comme un lieu unique. L'objectif 11 entend conforter un patrimoine naturel exceptionnel et une vitalité culturelle et sportive reconnue pour proposer une offre de loisirs toujours plus attractive.

Concernant la jeunesse (objectif 12), Le SRADDET de la Région CVL se donne un objectif qui peut paraître très général: "des jeunes épanouis et qui disposent des clés de la réussite pour préparer l'avenir" qui renvoie en fait à une forte augmentation du nombre d'étudiants (passer de 65000 actuellement à 80000 en 2030), à une forte diminution des jeunes sans diplôme, et à un rattrapage du retard de la proportion de jeunes avec qualification, par rapport à la moyenne nationale).

Pour ce qui est du développement économique et de l'activité agricole (objectifs 13 et 14), le SRADDET vise à atteindre $15 \%$ de surface agricole utile labellisée bio ou en cours de conversion en 2030 (2,3\% en 2015). Cette cible est ambitieuse et devra être couplée d'un accompagnement pour les exploitants propriétaires des $85 \%$ de surfaces agricoles restantes (en agriculture dite conventionnelle) qui doivent être aidés pour aller dans le sens d'une agriculture moins polluante ou raisonnée. Le SRADDET a également pour ambition de vouloir impulser et accompagner la transition économique et numérique de l'économie et des entreprises.

Enfin, l'objectif 15 relatif à l'accessibilité de la région, dont on peut estimer qu'il ne définit pas une véritable stratégie régionale en matière de transports, propose des mesures en faveur de l'équipement aéroportuaire régional ou de soutien aux projets ferroviaires ayant un impact de niveau régional.

L'orientation 4 - "Intégrer l'urgence climatique et environnementale et atteindre l'excellence écoresponsable" traduit un souci de forte prise en compte des impératifs écologiques et de réponse aux enjeux découlant du changement climatique (Objectifs 16 à 20).

Les ambitions affichées sont affirmées de façon résolue et forte :

- Concernant les questions énergétiques (objectif 16), sont définis des objectifs chiffrés par thématique et indiquées des étapes datées pour mesurer l'atteinte de ceux-ci. La Région souhaite accompagner la sortie progressive du nucléaire et aboutir à $100 \%$ d'énergies renouvelables et de récupération en 2050 alors que la Région est actuellement en France la première pour la production d'électricité d'origine nucléaire).

- Pour ce qui est de l'eau (objectif 17 : "l'eau, une richesse de l'humanité à préserver"), les préconisations retenues peuvent apparaître ambitieuses au vu du calendrier retenu et de l'état écologique actuel des cours d'eau. 
- Par ailleurs, en matière de biodiversité (objectif 18), l'ambition affichée n'est pas moins forte de faire de la Région CVL "la première région à biodiversité positive" en généralisant la réalisation d'inventaires de la biodiversité communale.

- Enfin concernant les déchets et l'économie circulaire "comme gisement de développement économique durable à conforter" (objectif 19 et 20 ), il faut noter la mise en place d'un observatoire régional des déchets et de l'économie circulaire et la volonté d'agir pour réduire et mieux valoriser les déchets et développer l'économie circulaire (réduction du gaspillage alimentaire de $50 \%$ en 2020 par rapport à 2013 et tendre vers $80 \%$ en 2031 ce qui est très ambitieux, réduire la production de déchets ménagers assimilés par rapport à 2010 de $10 \%$ en 2020 et de $15 \%$ en 2025, etc...).

Cette présentation très résumée des orientations et des objectifs pourrait donner à penser qu'il s'agit avant tout d'un catalogue de vœux pieux, mais il importe de souligner que le rapport les explicite de façon nettement plus précise en indiquant pour chacun d'eux des champs de mesures et des "cibles" quantifiées, et surtout en les complétant par les 47 règles générales incluses dans le "fascicule", que l'on ne peut présenter en détail dans le cadre limité de cet article. Ces règles, seules à être prescriptives comme on l'a vu plus haut, s'adressant aux plans et programmes locaux (SCoT, PLU(i), PDU, PCAET, Chartes des PNR, aux décisions morales de droit public et leurs concessionnaires dans le domaine de la prévention et la gestion des déchets...), concernent l'ensemble des territoires infra régionaux et toutes les collectivités locales de la Région. Elles s'inscrivent dans un rapport de compatibilité avec les documents de rang inférieur, c'est-à-dire que ceux-ci ne peuvent prendre de disposition allant à l'encontre ou remettant en cause ces règles générales.

L'architecture du fascicule s'articule autour de 5 thématiques : - équilibre du territoire (15 règles), - transports et mobilité (12 règles), - climat-air-énergie ( 8 règles), - biodiversité (5 règles), - déchets (7 règles).

Les règles sont toutes présentées selon le même cadre. L'élément prescriptif de la règle est identifié par un encadré permettant une meilleure appréhension par les différents acteurs ; mais des recommandations associées viennent compléter l'énoncé de la règle en enrichissant les préconisations. À titre de simple exemple, on peut évoquer la règle $\mathrm{n}^{\circ} 7$ : "Définir des objectifs de densité de logements pour les opérations d'aménagement, en renouvellement urbain comme en extension, le cas échéant». Cette règle stipule que les plans d'urbanisme doivent nécessairement inclure des objectifs de densité de logements à l'hectare pour les opérations d'aménagement ou d'extension. Elle précise le mode de calcul de la densité et indique que les objectifs de densité doivent prendre en compte les caractéristiques et la qualité patrimoniale, paysagère et naturelle des tissus urbains locaux et des formes urbaines. Pour autant, elle ne fixe 
pas de normes précises et intangibles de densité qui seraient à prendre en compte en tout lieu, pour permettre la meilleure adaptation possible de ces normes à la réalité locale. En revanche, dans les recommandations non prescriptives, des seuils de densités minimales et des fourchettes sont préconisées pour différents types de bâti (lotissements, grands ensembles, habitat intermédiaire, etc.). Les collectivités territoriales sont donc tenues d'imposer des normes de densité dans leurs plans locaux, mais leur marge de manœuvre reste large dans la définition de celles-ci, pour peu qu'elles respectent les orientations et objectifs du SRADDET, en particulier les objectifs 5 et 6 (endiguer la consommation d'espaces agricoles, naturels et forestiers dans la Région; renforcer l'accessibilité de l'habitat en tenant compte des changements sociétaux, climatiques et économiques.)

\subsection{Un schéma co-construit avec les acteurs locaux et les représentants de la société civile}

Le SRADDET de la région CVL, à l'image de ce qui se passe également dans la plupart des Régions françaises, résulte d'un processus de co-élaboration avec de nombreux acteurs, afin de concrétiser la volonté politique réitérée par l'exécutif régional d'aboutir à un projet partagé et durable d'aménagement et de développement $\mathrm{du}$ territoire. Il s'agit de déboucher sur des réponses aux enjeux d'équilibre et d'égalité des territoires, tels qu'ils se posent concrètement dans la Région, fondées sur des coopérations renforcées, des solidarités et des réciprocités entre territoires et acteurs locaux. En d'autres termes, la finalité de ce processus est de repenser la prescriptivité pour produire un SRADDET multi niveaux et aller vers une co-construction des objectifs et des règles, permise par un degré élevé de concertation et de négociation. Il s'agit d'adopter ensemble des normes plutôt que de les imposer. Telle est du moins l'ambition affichée pour rechercher les complémentarités entre métropoles et autres collectivités, entre territoires urbains et ruraux...On en espère une optimisation de la répartition des fonctions régionales entre l'ensemble des pôles urbains de différent niveau et une réorganisation des flux d'échange pour transcender la fragmentation régionale, et permettre à la Région de «faire système ».

C'est pourquoi, le rapport du SRADDET, dans sa version initiale, insiste sur le fait que : «le SRADDET est avant tout le résultat d'un travail collectif qui s'inscrit dans le droit fil de la démarche de concertation [...] et des principes de démocratie participative que la Région met en cuvre. [...] L'élaboration du SRADDET a mobilisé l'intelligence collective de tous, sous différentes formes entre novembre 2017 et juillet 2018. ».

De fait, 3400 participants ont été comptabilisés au cours des différents temps de rencontre et de travail, et 110 contributions écrites ont été rassemblées pour produire le rapport. En revanche, il ne semble pas que cette mobilisation ait été aussi importante au sein de la population : En témoigne le faible nombre de citoyens ayant renseigné le questionnaire mis sur le site démocratie-permanente.fr. Quoiqu'il en soit, 
la concertation a été élargie au-delà des acteurs prévus par la Loi. Un premier forum de lancement, très largement ouvert, a été organisé en novembre 2017. Des « ateliers $360^{\circ}$ ", sous forme de séances de brainstorming ont été organisés dans les 20 bassins de vie de la Région. Un « cercle des acteurs» réunissant les partenaires institutionnels de la Région a été mis en place pour permettre des échanges thématiques, en particulier sur la gestion des déchets, la mobilité, le climat, l'énergie, la biodiversité...Un espace de contribution a été ouvert en ligne sur le site web mentionné ci-dessus. En outre, en amont de cette concertation, le Conseil Économique, Social et Environnemental de la Région, assemblée représentative de la société civile organisée, rassemblant toutes les «forces vives » de la Région, a été saisi pour formuler des propositions qui ont pris la forme d'une «Contribution du CESER Centre-Val de Loire au SRADDET » (CESER, 2017). On peut observer une très forte congruence entre les recommandations déclinées dans cette contribution et le contenu du Schéma, ce qui laisse supposer que les avis de la société civile ont été considérés, à défaut d'être intégralement repris dans le rapport.

\section{Le SRADDET, un outil de développement territorial durable (DTD) ?}

On ne reviendra pas ici sur la définition du développement territorial durable (DTD), à laquelle nous avons consacré plusieurs écrits, y compris dans cette revue (Carrière, 2016, 2017, 2018) et deux ouvrages (Carrière et al. 2013 et 2016). En revanche, la question se pose de savoir si le SRADDET donne à la Région la capacité d'intervenir sur le terrain du DTD, alors que le schéma n'est pas encore formellement adopté dans aucune des Régions françaises. Les développements et analyses qui suivent sont totalement informés par cette interrogation.

La création du SRADDET marque un "retour" de la tradition de l'aménagement du territoire et de la planification territoriale, en France, ne serait-ce que par l'inclusion de l'égalité des territoires dans ses finalités, comme corollaire du développement durable. Il faut se souvenir que depuis ses origines, dans les années 1950, l'aménagement du territoire « à la française » a eu comme motivation principale et affichée la recherche d'un équilibre territorial du territoire français, en réponse au double problème de «l'hypertrophie parisienne » et des disparités Est-Ouest ${ }^{10}$. Nous ne reviendrons pas ici sur le bien-fondé de cette justification, et sur l'efficacité toute

\footnotetext{
10 On se souvient de l'ouvrage considéré comme fondateur de l'approche française de l'aménagement du territoire, "Paris et le désert français ", écrit par Jean Gravier en 1947, où l'auteur dénonçait à la fois l'hypertrophie de la Région parisienne (en termes à fois démographiques et économiques) et le déséquilibre structurel du territoire national, entre des Régions situées respectivement à l'ouest et à l'est d'une ligne Le Havre - Marseille, les premières accusant un retard de développement par rapport aux autres. Si le diagnostic peut paraître obsolète, aujourd'hui à l'époque de la métropolisation, force est de constater que la problématique des disparités régionales continue à structurer fortement la réflexion sur l'aménagement du territoire, en France.
}

L.S.G.D.C. 47 (1): $7-28$ 
relative de l'action publique au regard de cette double question. En revanche, il nous faut relever que le choix de l'intitulé du SRADDET est révélateur de la persistance d'une doxa officielle qui conduit à considérer l'Aménagement du Territoire comme une action publique destinée à réduire les disparités de développement entre les territoires ${ }^{11}$. Le SRADDET procède encore de cette vision.

Mais de toute évidence, le contexte est aujourd'hui fort différent par rapport à la période dite de «l'âge d'or » de l'aménagement du territoire en France, période correspondant peu ou prou aux débuts de la $5^{\text {ème }}$ République, sous la direction du pouvoir gaulliste. La globalisation, la crise économique et financière et l'affirmation des idées libérales ont été accompagnées par une forte réorientation des objectifs et des méthodes d'action en matière d'aménagement, en France comme dans le reste de l'Europe. En fait, si le SRADDET reste imprégné de la culture française de l'aménagement, il n'échappe pas aux tendances à l'œuvre en Europe, et relève in fine d'un compromis entre une tradition anglo-saxonne, foncièrement libérale, pour qui les documents d'aménagement ne sont que des "guidances», selon l'expression britannique, c'est-à-dire des documents de portée stratégique, flexibles et noncontraignants, porteurs d'une "Spatial vision», et une tradition française plus dirigiste, qui se concrétise par les notions de prescriptivité et d'opposabilité. Mais, on l'a vu, il s'agit d'une forme de prescriptivité, tout compte fait, peu coercitive, et le compromis s'établit plus en faveur de la tradition anglo-saxonne. On est loin de "l'ardente obligation » invoquée dans les années 60 par le Général de Gaulle pour caractériser le plan et l'aménagement du territoire. Par ailleurs, le constat s'impose : le SRADDET a été créé par les rédacteurs de la Loi NOTRe en affichant de grandes ambitions, à savoir : faire de la Région l'acteur central d'un développement équilibré et durable, tant sur le plan économique que social et environnemental. Qui plus est, il est supposé être l'un des instruments essentiels de la transition écologique, comme on peut le voir avec la place assignée à l'environnement dans les thèmes qu'il doit obligatoirement traiter. La Loi a suscité dans les Régions françaises une volonté forte de faire renaître l'aménagement régional, à l'image de la Nouvelle Aquitaine, qui a créé, en réorganisant ses services, un "Pôle DATAR », en charge de l'élaboration du Schéma. Cette Région, à l'instar de plusieurs autres, s'est donnée des moyens importants pour élaborer son SRADDET: Appel à de nombreux prestataires externes, création d'un comité scientifique du SRADDET, création d'un comité de pilotage auquel est complètement intégré le CESER en tant qu'organe représentatif de la «société civile organisée», lancement d'un grand nombre d'ateliers

11 Tout comme par ailleurs la transformation de l'ex-DATAR (Délégation à l'Aménagement du Territoire et à l'Action Régionale) en CGET (Commissariat Général à l'Égalité des Territoires) 
thématiques et territoriaux, avec exigence de synthèse, élaboration d'un diagnostic stratégique de la Région, précédant la définition des objectifs...

Mais le décalage reste considérable entre les ambitions et les capacités réelles des Régions, qui restent l'échelon le plus faible des pouvoirs locaux, ne serait-ce que parce que le budget consolidé des Régions françaises représente moins de $1 \%$ du PIB national! Ceci explique qu'elles aient tant de difficultés à peser sur les territoires infra-régionaux. Dès lors, on observe une distorsion entre d'une part des dépenses et des investissements régionaux bénéficiant plus aux espaces métropolitains (comme les investissements ferroviaires, la Région ayant dans ses compétences le transport régional) et d'autre part des SRADDET qui selon toute vraisemblance chercheront à promouvoir des actions de compensation en direction des territoires en difficulté, extra-métropolitains, ne parvenant pas à s'insérer dans le développement économique, en mobilisant des moyens somme toute modestes.

Alors, compte tenu de ces limites, on est fondé à se demander à quoi pourront aboutir les SRADDET ? Au regard des premières observations que nous pouvons relever à partir des premières esquisses régionales, le Schéma conduira à proposer à tous les acteurs une "vision spatiale » plus ou moins bien co-construite et partagée, avec les autres collectivités (communes, intercommunalités et Départements), mais aussi avec les Régions voisines ${ }^{12}$, et un diagnostic du développement régional (surtout dans les Régions à nouveau périmètre). Cette vision et le diagnostic qui la sous-tend pourront se révéler utiles pour que les territoires infra-régionaux élaborent eux-mêmes leurs propres stratégies, mais on est assez loin d'autres cas européens (Allemagne...) ou hors UE (Suisse) où se vérifie une véritable approche multiniveaux de la planification territoriale, et, où s'articulent mieux plans nationaux, régionaux et locaux. Or, un développement territorial durable à l'échelle régionale ne peut s'envisager sans une telle articulation, de façon à garantir la meilleure cohérence possible entre les aménagements ou les actions de développement local dans les territoires. Au total, la démarche générale adoptée par plusieurs Régions, à l'instar des Hauts de France, vise à permettre une fabrication partagée des règles et des objectifs, de façon à limiter la dimension prescriptive du SRADDET, et à en renforcer la mise en œuvre contractuelle : ainsi par exemple dans cette Région des Hauts de France, les territoires déjà dotés d'un SCoT sont invités à participer à l'ensemble des travaux préliminaires au SRADDET avant même d'avoir à donner leur avis sur le schéma final, comme la Loi l'impose. La finalité affichée de ce dispositif est de produire une "vision » régionale unique, partagée par toutes les collectivités, afin de permettre une meilleure distribution dans l'espace des grandes fonctions

12 Par exemple, la Région Hauts de France a invité les Régions frontalières des pays voisins (Flandres, Wallonie, Kent, et Luxembourg) à participer pour avis à l'élaboration de son SRADDET, tandis que la Région Centre - Val de Loire a sollicité ses Régions limitrophes. 
régionales avec le souci de penser la «mise en système du territoire régional », comme le disait un responsable régional des Hauts de France à l'occasion d'un séminaire européen organisé à Paris en avril $2018^{13}$; le même intervenant précisant alors : "Il ne s'agit pas d'imposer le schéma, mais de convaincre les territoires de la nécessité de l'approche multi-niveaux par l'articulation des différents niveaux de plans et de schémas ».

En second lieu, les SRADDET, dont le caractère réglementaire et prescriptif se révèle finalement beaucoup moins marqué que ce que l'on pouvait imaginer à la lecture de la Loi, vont produire des règlements et des normes qui ne s'appliquent qu'à quelques domaines (la régulation de la consommation d'espace, le traitement de la vacance commerciale...etc.). Si la concertation avec les autres niveaux de décision fonctionne bien, ces règles pourront s'imposer à tous. Par exemple, le SRADDET de la Région Centre - Val de Loire devrait, s'il est adopté, contenir des règles de consommation d'espace et de préservation des espaces non bâtis à intégrer dans les plans d'urbanisme locaux.

Enfin, les SRADDET auront à préciser les déclinaisons régionales des grands projets d'infrastructure et d'aménagement initiés par le niveau national.

Si ces différents effets ne sont pas à négliger, car permettant à la Région d'assumer son rôle de chef de file, on est encore loin d'une véritable approche multiniveaux à l'échelle du territoire régional, qui reste dans le meilleur des cas au stade de l'affichage d'intentions.

Il est sans doute prématuré de dresser un bilan, mais les premiers constats qui s'imposent à partir de l'observation des travaux d'élaboration dans les différentes Régions permettent de dégager quelques tendances générales. Il conviendra, bien entendu, de vérifier si elles se confirment à l'issue des processus de décision, fin 2019:

- Une comparaison rapide, à partir des informations auxquelles nous avons pu avoir accès de façon plus ou moins formelle selon les cas, laisse entrevoir une forte tendance au mimétisme dans les méthodologies d'élaboration et des premières orientations retenues, en dépit des spécificités géo-économiques des Régions; ce qui peut s'expliquer par l'importance prise dans la déclinaison des objectifs des schémas par l'importance donnée à des réponses localisées à des défis sociétaux et environnementaux universels (changement climatique, vieillissement des populations, raréfaction des ressources, menaces sur la biodiversité....)

- Les Régions rencontrent de réelles difficultés à assumer leur rôle de chef de file, en matière d'aménagement $d u$ territoire pour plusieurs raisons qui

13 Il s'agit d'un séminaire sur la planification régionale en Europe, organisé le 28 mars 2018 à l'Université Paris Diderot, par ESPON et le réseau de chercheurs RIATE 
tiennent à la fois aux difficultés à faire reconnaître leur légitimité à des collectivités locales, disposant parfois de moyens supérieurs aux leurs, comme c'est le cas avec les métropoles, et qui sont déjà dotées de leur propre projet de territoire. Il en résulte que la hiérarchie des documents de planification territoriale, voulue par la Loi, ne s'impose pas d'emblée.

\section{Premières conclusions}

La réponse effective qui sera apportée à la question posée dans le titre de cet article, "le SRADDET peut-il être un instrument de DTD, à l'échelle de la Région et de ses composantes territoriales (métropoles, villes, bassins de vie, territoires ruraux...) ?", dépendra de la façon dont le contenu et les modalités de leur mise en œuvre des schémas seront définis.

En premier lieu, le DTD implique une cohérence de l'action publique garantissant la compatibilité des objectifs et des actions mises en œuvre. Or cette cohérence ne peut être obtenue sans une véritable articulation des différents niveaux de plans et de schémas. En d'autres termes, condition nécessaire, mais non suffisante, la planification régionale doit procéder d'une véritable approche multi niveaux.

En second lieu, le SRADDET ne pourra respecter ses finalités, l'aménagement et le développement durables, et l'égalité des territoires, que s'il est conçu en sortant d'une logique compensatoire et réparatrice et inscrit dans ses priorités la valorisation des potentialités des territoires non métropolitains, en favorisant une dynamique de développement endogène, à l'échelle de chacune des composantes du territoire régional.

Mais le succès du SRADDET requiert aussi de sortir des approches courttermistes. Le DTD s'inscrit dans un temps long qui n'est pas celui du mandat politique.

Enfin, le respect des pré-requis d'une véritable démocratie participative est une nécessité incontournable pour permettre à l'ensemble des acteurs et à la population de contribuer à la définition des orientations et à la réalisation des objectifs du Schéma.

\section{ORCID}

Jean-Paul Carrière @ 0000-0002-5781-4369 


\section{Références}

Carrière J-P. (2016). La requalification des friches urbaines : enjeu et outil des politiques régionales de développement territorial durable. Lucrările Seminarului Geografic "Dimitrie Cantemir » $43: 5-24$.

Carrière J-P. (2017). Les défis du développement territorial durable pour la formation des aménageurs - urbanistes : quelques questions introductives à partir des innovations du Département Aménagement et Environnement de Polytech'Tours, Lucrările Seminarului Geografic « Dimitrie Cantemir » $44: 15-26$.

Carrière J-P. (2018). La région, une échelle pertinente pour la « mise en territoire » de l'économie circulaire? réflexions à partir du cas français. Lucrările Seminarului Geografic "Dimitrie Cantemir $\gg 46: 3-23$.

Carrière J-P., Demazière C., Petrea R. Filimon L. (2013), La mise en œuvre du développement territorial durable. Déclinaisons franco-roumaines. Ed. L'Harmattan, Paris, $276 \mathrm{p}$.

Carrière J-P., Hamdouch A., Iațu C. (2016). Développement durable des territoires. Ed. Economica Anthropos, Paris, $234 \mathrm{p}$.

CESER Centre Val de Loire. (2017). Contribution du CESER Centre-Val de Loire au SRADDET : 27 chantiers pour doper l'attractivité régionale et répondre aux besoins de la population, 101 p.

Howard E. (1898). Tomorrow : A Peaceful Path to Real Reform. Traduit en français sous le titre Les cités jardins de demain. Dunod 1969, coll. Aspects de l'urbanisme.

Région Centre - Val de Loire. (2018a). Synthèse du projet de SRADDET, $12 \mathrm{p}$.

Région Centre - Val de Loire. (2018b). La région 360. SRADDET Centre - Val de Loire. Version pour arrêt de décembre 2018, 254 p.

Torre A., Bourdin S. (2015), Le big bang territorial. La réforme des régions en débat. ED. Armand Colin, Paris. 
Annexe 1. Les compétences des Régions françaises d'après la Loi NOTRe

En plus des compétences qu'elles partagent avec les Départements et les communes (tourisme, sport, culture, éducation populaire, lutte contre la fracture numérique...), les Régions, qui ne bénéficient plus de la clause de compétence générale contrairement aux communes, jouissent désormais de prérogatives exclusives, qui déterminent la répartition de leur budget propre. Elles exercent leurs compétences dans les domaines suivants :

- Les transports : devenues autorités organisatrices de transport de plein exercice, elles gèrent des ports et aéroports, les trains express régionaux, des transports routiers interurbains et scolaires, les gares publiques routières...

- Les lycées : construction et entretien des lycées d'enseignement général ou agricole...

- La formation professionnelle : insertion des jeunes en difficulté, formation des demandeurs d'emplois, gestion de l'apprentissage et des formations en alternance...

- Le développement économique : animation des pôles de compétitivité, aides au tissu économique et aux entreprises, mise en œuvre du Schéma Régional de Développement Economique, d'innovation et d'internationalisation (SRDE-II) qui définit les orientations en matière d'aides aux entreprises, de soutien à l'internationalisation, d'aides à l'investissement immobilier et à l'innovation des entreprises. Ce schéma doit être en cohérence avec le SRADDET.

- La gestion des programmes européens, la Région étant devenue autorité de gestion des fonds structurels de l'UE sur son territoire.

- $\quad$ Et enfin, l'aménagement du territoire et l'environnement, la Région devant assurer la gestion des déchets, des parcs naturels régionaux, et étant responsable de l'action publique en matière de développement rural et urbain ou de préservation de la qualité de l'air... Elle est aussi en charge du SRADDET, comme on l'a vu dans cet article. 